
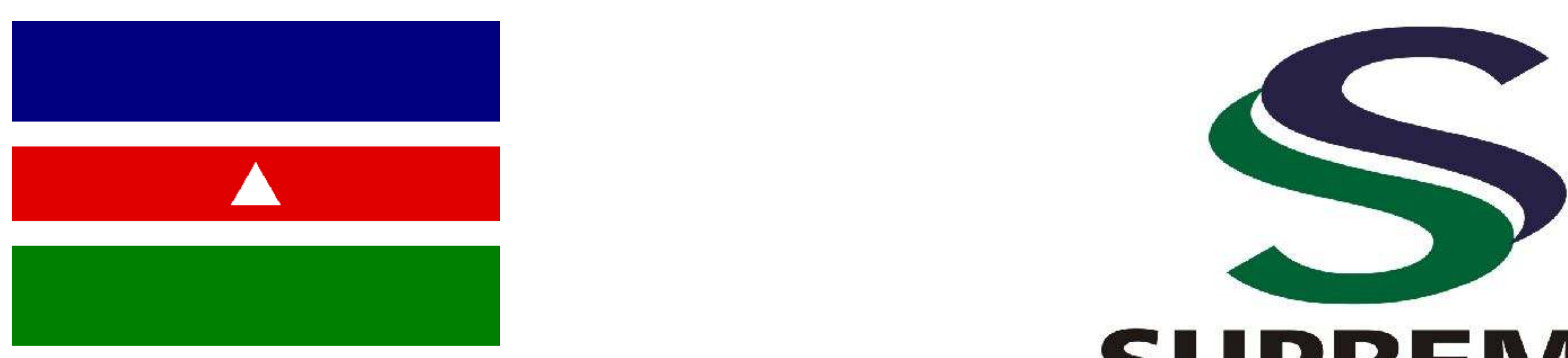

SUPREMA

de Juiz de Fora

\title{
Diabetes Mellitus Type I and it's Relationship With Periodontal Disease: A Systematic Review
}

\author{
OLIVEIRA, R.G., PICININI, L.S., GOMES, M.M., FERREIRA, A.P., COSTA, J.F.P., SANTANA, R.B.
}

\section{BACKGROUND}

The prevalence, severity and progression of periodontal disease (PD) is related to poor glycemic control in type 1 diabetic patients (DM1), however, contradictory results are reported with regard to the influence of PD on metabolic control in patients who depend on insulin. Thus, our goal was to review the scientific evidence that establishes a link between PD and DM1, analysing the possible metabolic influences for the development and worsening of these ailments, as well as possibly identifying courses of intervention that could favour control, or reduce the influence of one disease on the other.

\section{MATERIALS AND METHODS}

This review is prepared in accordance with the PRISMA (Preferred Reporting Items for Systematic Reviews and Meta-Analyses) statement based on the proposed focused questions. A literature search with no restrictions regarding status or the language of publication was performed for MEDLINE and EMBASE databases up to and including December 2016. Systematic reviews (SRs), randomized clinical trials, and controlled clinical trials, evaluating the influence of DP in DM.

\section{RESULTS}

Of the 423 potentially eligible trials, 4 were included and data were assessed.

Figure 1. Study flow diagram.

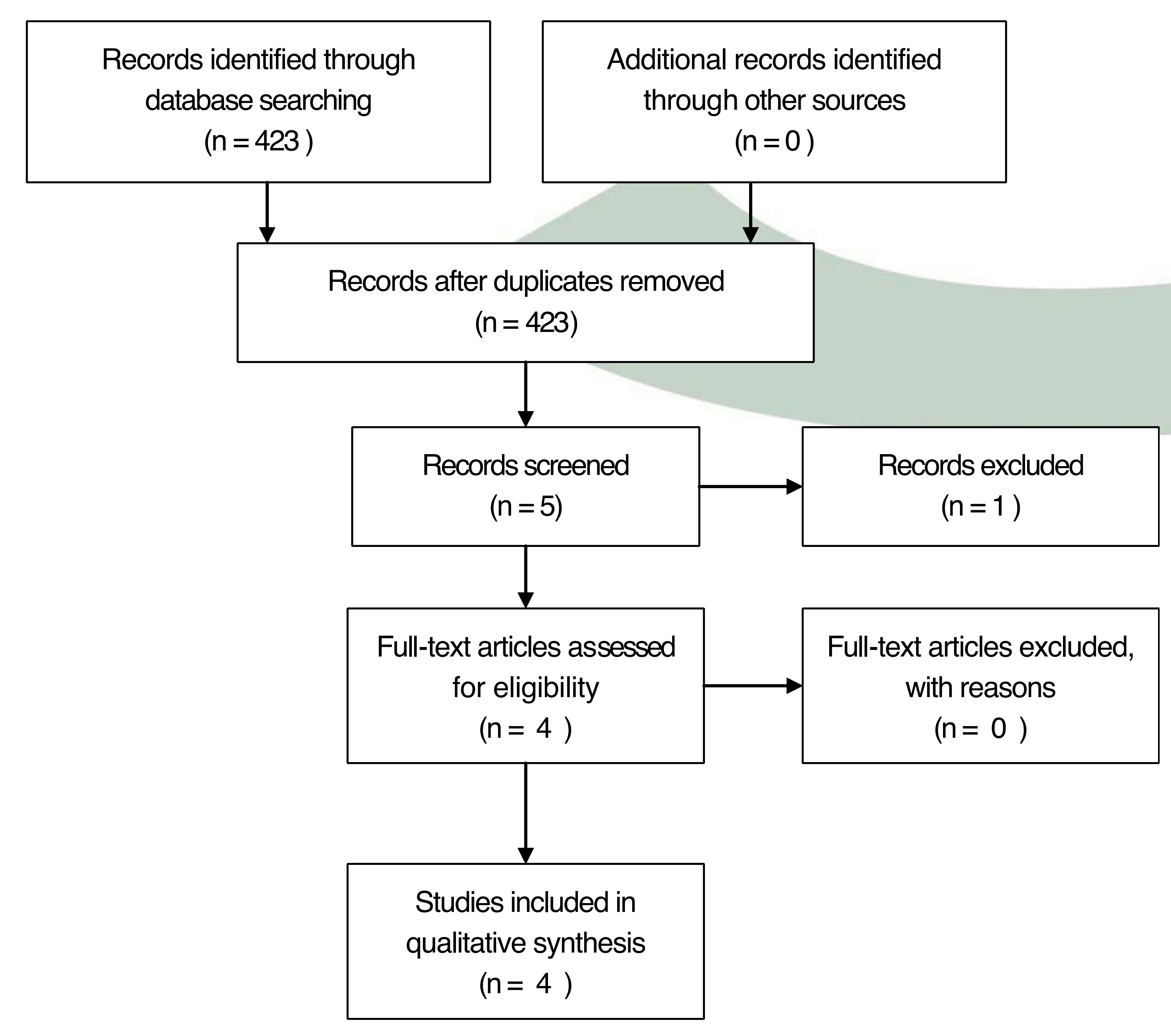

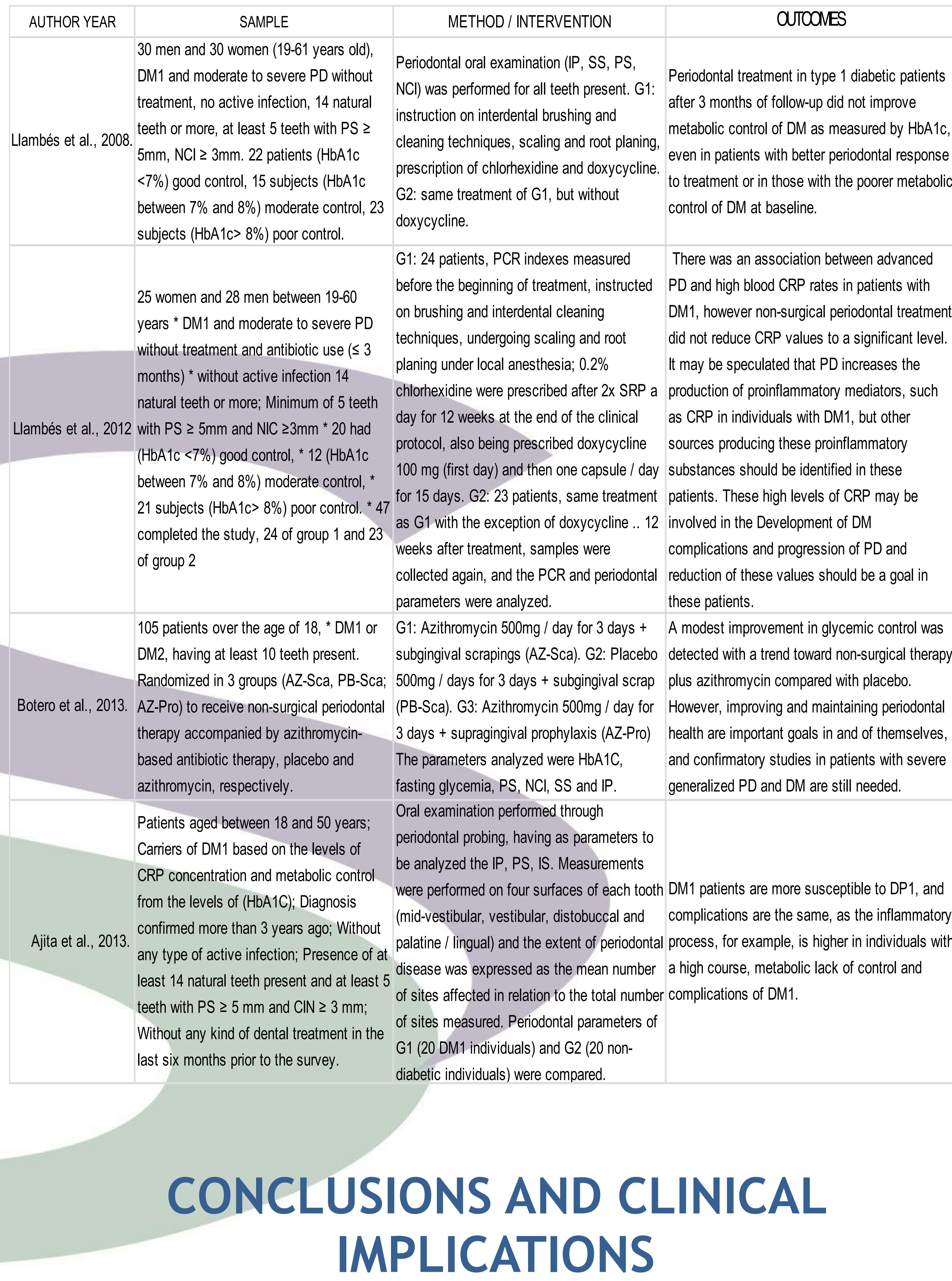

AUTHOR YEAR 30 men and 30 women (19-61 years old), DM1 and moderate to severe PD without treatment, no active infection, 14 natural Llambés et al., 2008. teeth or more, at least 5 teeth with PS $\geq$ $5 \mathrm{~mm}, \mathrm{NCl} \geq 3 \mathrm{~mm}$. 22 patients (HbA1C $<7 \%$ ) good control, 15 subjects (HbA1C between $7 \%$ and $8 \%$ ) moderate control, 23 subjects (HbA1c> 8\%) poor control.

25 women and 28 men between 19-60 years ${ }^{*} \mathrm{DM} 1$ and moderate to severe $\mathrm{PD}$ without treatment and antibiotic use ( $\leq 3$ months) * without active infection 14 natural teeth or more; Minimum of 5 teeth Llambés et al., 2012 with PS $\geq 5 \mathrm{~mm}$ and NIC $\geq 3 \mathrm{~mm} * 20$ had (HbA1C $<7 \%$ ) good control, * 12 (HbA1C between $7 \%$ and $8 \%$ ) moderate control, 21 subjects (HbA1c> 8\%) poor control. * completed the study, 24 of group 1 and 23 of group 2

105 patients over the age of $18,{ }^{*} \mathrm{DM} 1$ or DM2, having at least 10 teeth present. Randomized in 3 groups (AZ-Sca, PB-Sca; 500mg / days for 3 days + subgingival scrap plus azithromycin compared with placebo.

Botero et al., 2013. AZ-Pro) to receive non-surgical periodontal (PB-Sca). G3: Azithromycin 500mg / day for However, improving and maintaining periodontal therapy accompanied by azithromycin- $\quad 3$ days + supragingival prophylaxis (AZ-Pro) health are important goals in and of themselves, based antibiotic therapy, placebo and The parameters analyzed were HBA1C, and confirmatory studies in patients with severe azithromycin, respectively. fasting glycemia, $\mathrm{PS}, \mathrm{NCl}, \mathrm{SS}$ and IP. generalized PD and DM are still needed. Patients aged between 18 and 50 years; Oral examination performed through Carriers of DM1 based on the levels of periodontal probing, having as parameters to CRP CRP concentration and metabolic control were performed on four surfaces of each tooth DM1 patients are more susceptible to DP1, and from the levels of (HDA1C); Diagnosis confirmed more than 3 years ago; Without any type of active infection; Presence of at least 14 natural teeth present and at least 5 of teeth with $P S \geq 5 \mathrm{~mm}$ and $\mathrm{CIN} \geq 3 \mathrm{~mm}$; Without any kind of dental treatment in the last six months prior to the survey. were performed on four surfaces of each tooth DM1 patients are more susceptible to DP1, and
(mid-vestibular, vestibular, distobuccal and complications are the same, as the inflammatory palatine / lingual) and the extent of periodontal process, for example, is higher in individuals with disease was expressed as the mean number a high course, metabolic lack of control and of sites affected in relation to the total number complications of DM1. of sites measured. Periodontal parameters of G1 (20 DM1 individuals) and G2 (20 nondiabetic individuals) were compared.

\section{CONCLUSIONS AND CLINICAL} IMPLICATIONS

PD is associated with higher levels of uncontrolled glycemic levels in patients with DM1. Periodontal interventions aimed at controlling bacterial and periodontal inflammation may positively influence the control of blood sugar levels and levels of inflammatory protein. 